\title{
2685. Fluid-solid coupling field analysis of centrifugal fan based on nonlinear dynamics
}

\author{
Chenxu Luo ${ }^{1}$, Shuangxi Jing ${ }^{2}$, Xiaoming Han ${ }^{3}$, Junfa Leng ${ }^{4}$ \\ School of Mechanical and Power Engineering, Henan Polytechnic University, Jiaozuo, 454000, P. R. China \\ ${ }^{1}$ Corresponding author \\ E-mail: 1'cumtlchx@hpu.edu.cn, ${ }^{2} j x x 196206 @ 163 . c o m,{ }^{3}$ hanxm@hpu.edu.cn, ${ }^{4}$ lengjunfa@163.com
}

Received 1 June 2017; received in revised form 5 August 2017; accepted 19 September 2017 DOI https://doi.org/10.21595/jve.2017.18705

Check for updates

\begin{abstract}
According to the characteristics of Fluid-solid coupling flow field of centrifugal fan, fan aerodynamic performance signal collecting experiment under different conditions are carried out on the centrifugal fan test bed. The relationship among airflow pressure pulsation, noise and vibration are studied by using nonlinear dynamics methods such as phase space reconstruction, correlation dimension, and complexity and so on, which lays the foundation for predicting the fan running status by monitoring the signal of airflow pressure pulsation and the noise. The result shows that the phase diagram of airflow pressure pulsation signal attractor is the most distinct and regular among the three signals. The scope of the phase diagram increases and becomes sparse and confusing with the decrease of the flow, which reflects that pressure pulsation amplitude becomes larger and the internal flow deteriorates gradually with the decrease of flow. The correlation dimensions are calculated with G-P algorithm of the three signals first decrease and then increase with the decrease of the flow. The correlation dimension of the airflow pressure pulsation or noise under stall operating condition can be used as monitoring indicators. The L-Z complexity of the noise increases constantly with the decrease of the flow. But the L-Z complexity of airflow pressure pulsation and vibration first decreases and then increases with the decrease of the flow.
\end{abstract}

Keywords: centrifugal fan, fluid-solid coupling, vibration, noise, correlation dimension.

\section{Introduction}

Centrifugal fan play an important role in the coal mine production. As the main source of power for coal mine ventilation, centrifugal fan can not only ensure the security of coal production, but also reduce probability of the occurrence coal mine gas accident effectively. The research on the dynamic behavior of centrifugal fan has great significance in aspect of improving production security and ensuring the safety of the personnel, because once the fan fails, serious economic loss and personnel safety accident will take place. As an important branch of fluid-solid coupling aerodynamic-elastic, impeller machinery belongs to fluid-solid coupling problem. It is very important to study the internal and external coupling of the fan in the aspect of improving fan impeller performance, structural strength, vibration characteristics, safety of fan operation reliability and the fan ability of prolonging working life. The paper [1] analyzes the shortcomings of mine centrifugal fan, and puts forward some measures of improving the safety of coal mine and preventing the ventilation accident. The paper [2] indicates that the source of initial cracks on the blade was corrosion and crack propagation, and pitting on the surface of the blade were controlled by a high cycle fatigue process. The essay [3] indicates that the crack in the blade was initiated by fretting fatigue and spread under low cycle fatigue by analyzing the fracture surface of the failed blade. The essay [4] studies the problem of Fluid-solid interaction (FSI) of turbo-machinery. The literature [5] introduces treatment scheme with the basic theory of fluid structure interaction, and summarizes the interaction, with experiment, numerical methods of the realization of turbine machinery and fluid structure. Chinese scientific research workers Li Jiafeng, Wang Jun, Li Hui who specialize in wing and aero engine, have done a lot of work to lay the foundation for the development of Chinese aero elastic theory in the aspect of aero-engine aero-elastic in the literature [6]. Hy Cui, N. Ding and M. Hong analyze the flow field and structural characteristics 
of the fan by using modern numerical simulation methods. pick out finite element method which based on computational fluid dynamics (CFD) and computational structural dynamics (CSD) comprehensively in the literature [7]. In the literature [8], Ata I. researches the methods of blade flutter and progress of the study model, by comparing the advantages to disadvantages of different methods, of which dealing with blade flutter. In the literature [9], Salhi B. and Lardies Jet introduce a method of analyzing the vibration data from a limited number of measurements. In the literature [10], the impeller fluid solid coupling analysis and fatigue life analysis are completed under the aerodynamic force, the centrifugal force and the gravity effects of the blade. Literature [11] summaries research status at home and abroad in fields of blade tip-timing sensing, blade vibration and damage monitoring, and the paper indicates that the leading edge and trailing edge of the blade which closed to the blade tip have obvious vibration problems with wave vibration dominant, so stiffness allowance should be considered fully in fan designed. In the literature [12], according to the aerodynamic characteristics of fan blades and the basic theory of fluid-solid coupling, the aerodynamic elastic analysis of fan blades was carried out, the G-P algorithm, the calculation method of the correlation dimension of the diesel engine sound signal are expounded, and the influence of the noise on the correlation dimension in the calculation process is discussed in detail. The paper [13] uses the energy method to predict the blade flutter of modern gas-turbine engines, and the correlation dimension to stall signal can be used as characteristic quantity to judge the rotating stall. The paper [14] indicates that non-contact measurement method is install easily and has no interference by measured blade. Literature [15] gets the analysis of the tip clearance change rule by adopting the thermal structure coupling technology for different operation conditions of the turbine blade tip clearance change simulation study. In the literature [16], a cavitation monitoring system of hydraulic turbines is developed based on multidimensional cavitation analysis and full-wave rectified spectrum analysis method to extract cavitation characteristic signals. In the literature [17], the vibration signals of axial impeller are analyzed and flow fluctuation are collected in different condition by correlation dimension, approximate entropy and L-Z complexity. Zhang Xinming sums up the shortcomings of the current research from domestic and foreign in the perspective of tip timing sensor, blade vibration and damage monitoring in the literature [18]. The paper [19] indicates chaotic motion can be used to detect and diagnose the early fault signal of the rotating machine by the phase change of the chaotic motion, which can provide an effective basis for the early fault diagnosis of the rotating machinery in engineering practice. The literature [20] studies the sensitivity of the chaotic system to the immunity to the noise, so that the chaotic system can detect the weak periodic signal in the noise background. The literature [21] indicates that the flutter frequency is close to the natural frequency of the blade, with the inlet speed and the angle of attack are important factors that affect the stability of the air elasticity. The literature [22] predicts the vibrations during blade processing. For the interaction of wind and flexible vanes, literature [23] uses a non-matching interface discretization method in which aerial dynamics are calculated using alevms based on low-order finite element, while rotor blades are modeled using nurbs-plasma analysis. In literature [24], a structural analysis method is proposed to solve the problem of large Fluid-solid interaction. The literature [25] points out stabilize the operating conditions, the main frequency of the impeller around the same pressure fluctuations through the same blade. The literature [26] uses the finite element method to calculate the dynamic response of the volute One-way coupling from fluid to structure. According to the basic theory of acoustics, Literature [27] determines the location and noise type of the main aerodynamic noise source inside the fan.

By taking Centrifugal fan impeller mechanism as the research object, this topic explores the relationship between aerodynamic pulsation and leaf structure, the mechanism of fan blade failure based on the characteristics of fluid-solid coupling flow field and impeller-fluid-solid coupling. By investigating the relationship of flow pulsation-noise-vibration in flow field and monitoring blade vibration and noise, the paper put forward the possibility of predicting the operating state of the fan and working condition of blade. The methods of control impeller mechanical fluid-solid coupling vibration, which using noise to diagnose the fault and improving the safety and reliability 
of impeller mechanical operation are raised to achieving the comprehensive management of impeller mechanical failure.

\section{Experimental study}

Based on the similarity theory, the experimental platform which simulates aerodynamic performance of Centrifugal fan, include the vibration, airflow pulsation and noise measuring point of the model are displayed in Fig. 1.

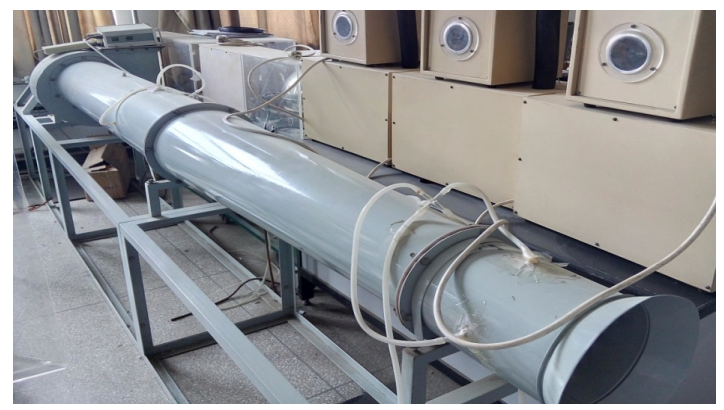

Fig. 1. Experimental platform for mechanical fault diagnosis of Centrifugal fan

The acceleration sensor, gas pressure sensor, microphone are used to measure the vibration signal, airflow pulsation signal, noise signal. B\&K dynamic signal acquisition and analysis system are used to measure the vibration signal, airflow pulsation signal, noise signal. The output signal of sensors are converted into a digital signal by a collector after being processed by a preamplifier or a front conditioner. The measuring instrument system integrates gain, filter, multiplexer, sample hold circuit, A/D converter, memory and logic control circuit. A good foundation for the experiment are provided by signal acquisition and conversion system which consists of collector, pressure sensor, front conditioner and signal amplifier. The whole system working principle is shown in Fig. 2.

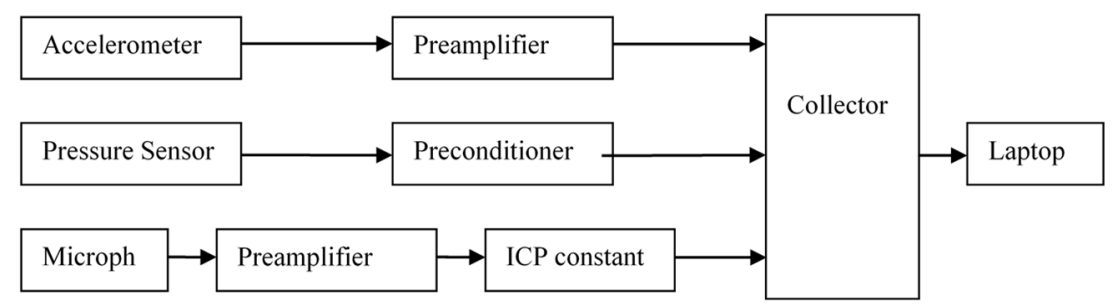

Fig. 2. Sketch of signal measurement system working principle

Measurement of contact with the strain gauge for measuring the vibration of the blade has shortcomings, such as difficult in installation, high cost and difficult to achieve. The sensors of this method which are mounted on a relatively stationary housing to meet the requirements of synchronization with the pulsating pulse, because vibration signal can be obtained only when the blade rotate to a specific position. The indirect measurement scheme, which taken in this paper meets the requirements of signals. Pressure sensors among different levels and microphones are installed to measure airflow pulsation and fan noise. The fan axial vibration is reflection of entire flow field because the rigid connection of blade and the hub make blade vibration is bound to reflect on Impeller axial vibration. The pressure sensors installed between the stages measure airflow pulsation. The microphone sensors measure fan noise.

Since the analysis of collected signals is in the help of computer or an analytical instrument, the continuous signals are discarded. According to Nyquist sampling theorem, we set the sampling 
frequency $5120 \mathrm{~Hz}$, number of sampling blocks 32, each block 1024 points, each group of acquisition time $6 \mathrm{~s}$, to meet the needs of data preprocessing anti-mixing and digital filtering. And according to the actual situation, the gain and amplification of the channel system are set separately. The signals of the three channels are collected at the same time.

\section{Data processing}

In the main measurement the normal flow of the fan is defined as $\Phi=0.770$. The abnormal flow of the fan is defined as Malfunction $1 \Phi=0.605$, Malfunction $2 \Phi=0.479$, Malfunction 3 $\Phi=0.228$.

1) Changing the flow of the fan, axial vibration signal under normal flow and abnormal flow will be measured by acceleration sensor and entered into the acquisition device. (2) The pressures among different levels flows under the normal or abnormal traffic, are measured by dynamic pressure sensor and entered to the acquisition device. (3) According to the standard of ventilator noise test, the noise of two different working conditions is collected by the microphone, and entered acquisition device after being detected. Time-domain signals of vibration, flow pulsation, and noise under normal and abnormal flow rates are collected by collection equipment and shown in Fig. 3.

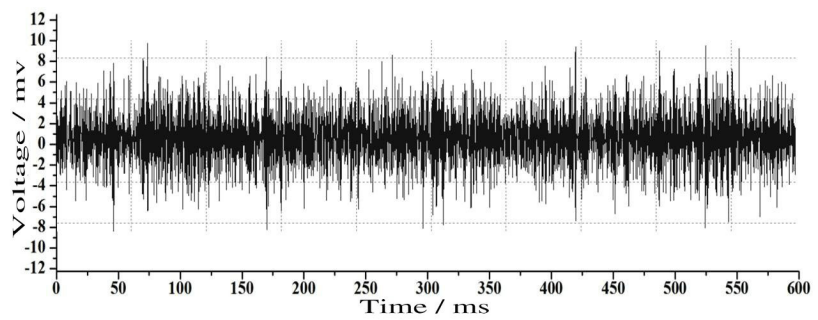

a) Time-domain signals of vibration under normal

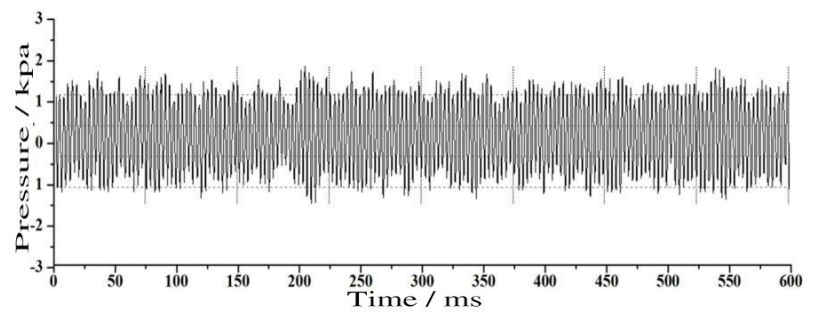

b) Time-domain signals of flow pulsation under normal

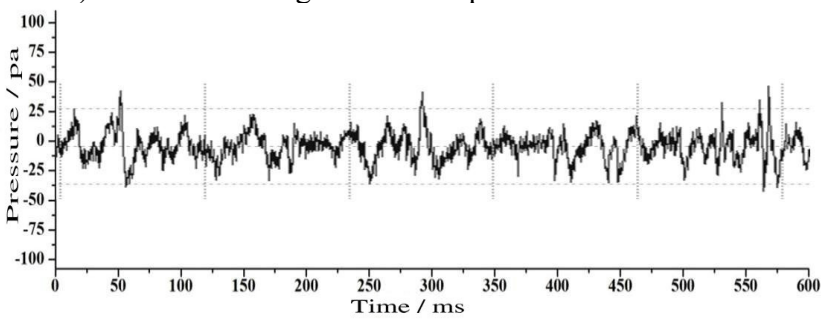

c) Time-domain signals of noise under normal

Fig. 3. Time-domain signals

As shown in the Figs. 4, 5, the strange attractors of flow pulsation, noise, vibration under flow changes tend to be more complex and intensity reduced. Two strange attractors appearing from fault, indicate that existence of failure in chaotic system will lead to the change of strange attractor. The attractor spectrum of the pulsating signal with the least noise is the most clearly and regularly. The increasing range of phase diagram change into sparse and chaotic as the flow decreases. From 
which can be reflected the decrease in the flow rate will cause increase of airflow pulsation, and deterioration of internal flow of the ventilator.

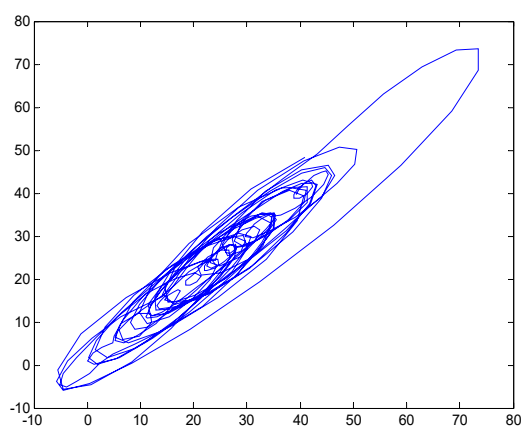

a)

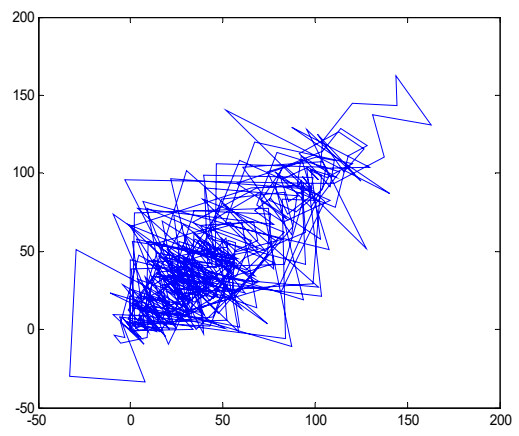

b)

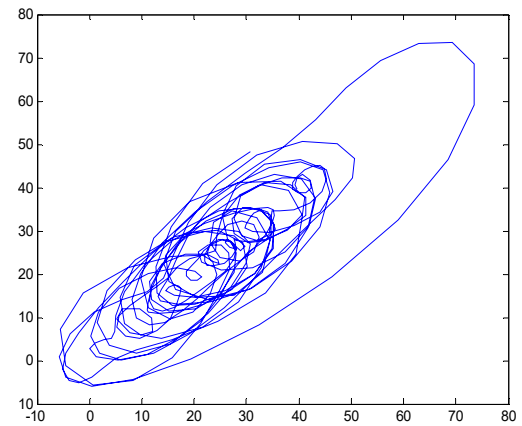

c)

Fig. 4. Normal flow signal phase space reconstruction

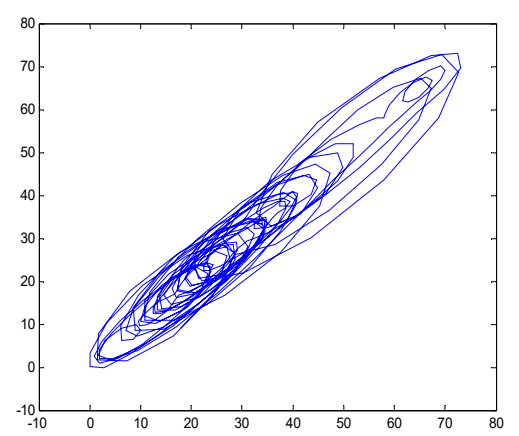

a)

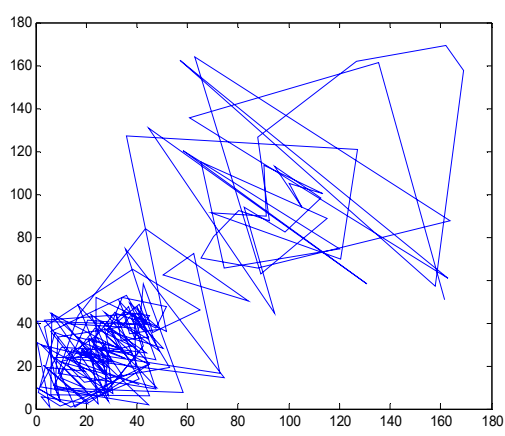

b)

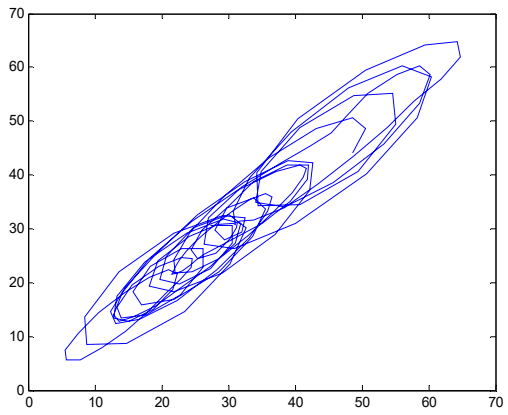

c)

Fig. 5. Graphic fault flow signal phase space reconstruction 
By observing noise signals and vibration signals, with less attracting features, but it can still be seen that the range of phase diagrams increases and becomes sparse and chaotic as the flow decreases, with the flow reduce, the noise of the fan becomes larger, the vibration becomes violent. This means that the three attractors have the same characteristics and reflect the operating state of the fan to a certain extent. It can be seen from the figure that the phase diagram of the airflow pulsation and the noise collection signal of the ventilator are disordered due to the influence of the fault signal, and the signal after being de-noised by wavelet shows clear and regular chaotic attractor characteristics. As more information contained, geometric characteristics of vibration signal attractor which before and after de-noising are not obvious, but its attractiveness has improved.

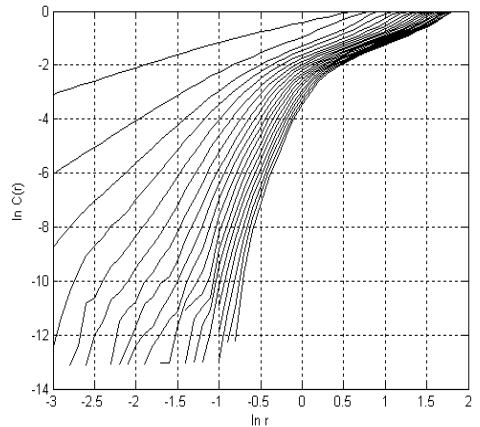

a) Associated point

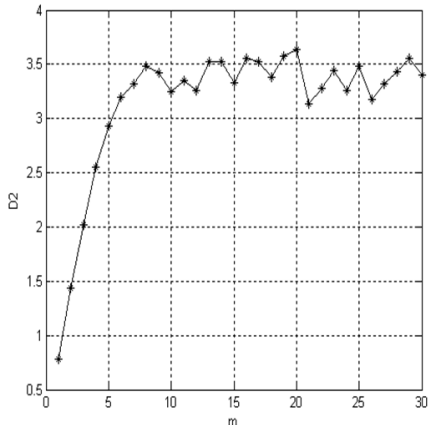

b) Association index and embedding index

Fig. 6. Correlation dimensions of flow pulsation under condition $\Phi=0.770$

As shown in Fig. 3-6, the correlation exponent increases with the increase of the embedded dimension $m$ and reaches saturation at $m=13$. The correlation dimension of airflow pulsation is 0.3691 under working conditions $\Phi=0.770$. This shows that the pulsation of the air flow has certain chaotic characteristics and wavelet de-noising has a certain de-noising effect on the pulsation signal.

Table 1. Correlation dimension of the airflow pulsation, noise and vibration

\begin{tabular}{|c|c|c|c|}
\hline & Airflow pulsation $\left(10^{1}\right)$ & Noise $\left(10^{1}\right)$ & Vibration $\left(10^{1}\right)$ \\
\hline $\begin{array}{c}\text { Normal } \\
\Phi=0.770\end{array}$ & 0.3691 & 0.4336 & 0.7090 \\
\hline $\begin{array}{c}\text { Malfunction 1 } \\
\Phi=0.605\end{array}$ & 0.3867 & 0.5068 & 0.7559 \\
\hline $\begin{array}{c}\text { Malfunction 2 } \\
\Phi=0.479\end{array}$ & 0.4165 & 0.5859 & 0.8174 \\
\hline $\begin{array}{c}\text { Malfunction 3 } \\
\Phi=0.228\end{array}$ & 0.4277 & 0.6299 & 0.8375 \\
\hline
\end{tabular}

The best embedding dimension $m$ and correlation dimension of the airflow pulsation, noise and vibration de-noising signals of the ventilator under various conditions are shown in Table 1. It can be seen from the Fig. 7 that the correlation dimension of airflow pulsation, noise and vibration are interrelated and have a tendency of decrease first and then increase with the decreasing trend of flow rate, and the pulsating signal and fan noise signal of the airflow can reflect the operating state of the fan and working state of the blade to a certain extent. The numerical simulation results show under the working condition $\Phi=0.605$, Fan has a slight rotation stall, the rotation stall will become serious, with the flow reduction, and there are a large difference in the aspect of associated dimension between the condition of stable operation with rotation stall. Under the working condition $\Phi=0.479$, the ripple dimension of airflow pulsation or noise can be used as operating state of the fan and the blade monitoring index. Axial fan works 
in the rotational stall working condition, in which the air flow pulsation signal acquisition process is less likely to be disturbed.

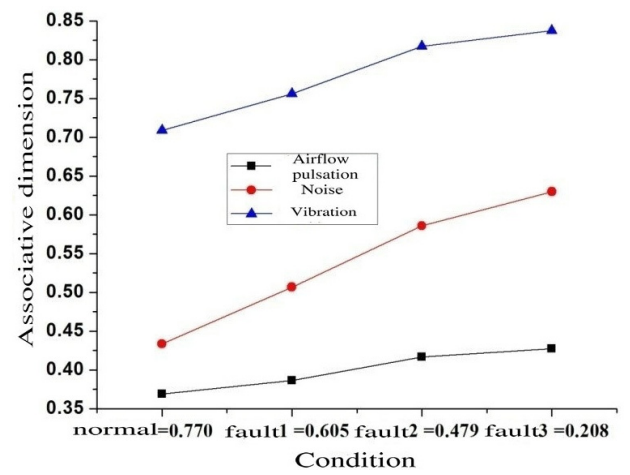

Fig. 7. Correlation graph of associative dimension under different working conditions (101)

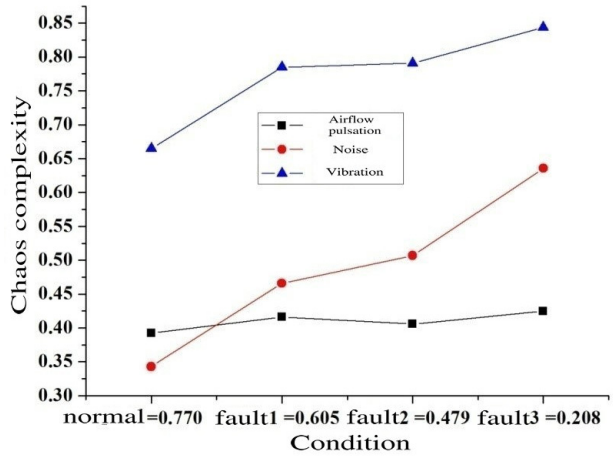

Fig. 8. Chaotic complexity results diagram under different operating conditions

Table 2. Complexity calculation results of condition

\begin{tabular}{|c|c|c|c|}
\hline & Airflow pulsation & Noise & Vibration \\
\hline $\begin{array}{c}\text { Normal } \\
\Phi=0.770\end{array}$ & 0.3926 & 0.3428 & 0.6648 \\
\hline $\begin{array}{c}\text { Malfunction 1 } \\
\Phi=0.605\end{array}$ & 0.416 & 0.4658 & 0.7847 \\
\hline $\begin{array}{c}\text { Malfunction 2 } \\
\Phi=0.479\end{array}$ & 0.4058 & 0.5068 & 0.7910 \\
\hline $\begin{array}{c}\text { Malfunction 3 } \\
\Phi=0.208\end{array}$ & 0.4248 & 0.6357 & 0.8438 \\
\hline
\end{tabular}

It can be seen from the Fig. 8 that if large flow condition is ignored, the complexity of airflow pulsation, noise and vibration are consistent and the complexity of the pulsating and vibration are consistent, and the decrease of the flow decreases with the decrease of the flow. Airflow pulsation, noise and vibration are interconnected, the air flow pulse signal and the fan noise signal can reflect the operating state of the fan and the certain working state of the blade. It is more feasible to predict the running state of the fan and the state of the blade work by monitoring the signal of air flow pulse.

\section{Conclusions}

By reconstructing the phase space of the three attractors under different conditions, the attractor tends to be complicated and the intensity tends to decrease with the change of flow. The attractor spectrum of the pulsating signal is the most clearly and regularly. The phase diagram becomes range increases sparse, confusing as the flow decreases. The decrease of the flow rate will cause increase of airflow pulsation, and deteriorate of internal flow of the ventilator.

Calculated by using the GP algorithm, with the decrease of the flow rate, the correlation dimension of three signals shows a tendency of decrease firstly and then increase. The correlation of the airflow pulsation or noise in the stall condition can be taken as a fan stall monitoring indicators.

By calculating the L-Z complexity of the three signals under different working conditions, this shows that that the complexity of noise increases with the decrease of flow rate, and the complexity of airflow pulsation and vibration decreases with the decrease of flow rate. It is more feasible to predict the operating state of the fan and the state of the blade by monitoring the airflow pulsation signal. 
So as to lay the foundation of predicting the operating state of the fan and the blade working conditions by monitoring the fan flow pulsation and noise signals. So, it is more feasible to predict the fan running status and leaves state by monitoring the signal of airflow pressure pulsation.

\section{Acknowledgements}

The authors are grateful for the funding of this work by the Henan Province Education Department Applied Research Project Fund (16A460005) and Henan Polytechnic University Doctor Research Project Fund (660407/020) and China Postdoctoral Science Foundation Funded Project (Project No. 2016M592289).

\section{References}

[1] Xue Xiaomin Management of coal mine safety ventilation and prevention measures of ventilation accident. Inner Mongolia Coal Economy, Vol. 5, 2013, p. 157-160.

[2] Poursaeidi E., Babaei A., Behrouzshad F., et al. Failure analysis of an axial compressor first row rotating blades. Engineering Failure Analysis, Vol. 28, Issue 2, 2013, p. 25-33.

[3] Lee B. W., Suh J., Lee H., et al. Investigations on fretting fatigue in aircraft engine compressor blade. Engineering Failure Analysis, Vol. 18, Issue 7, 2011, p. 1900-1908.

[4] Zhu Fengmei The Analysis of Fluid-Solid Interaction of the Large Centrifugal Fan and the Vibration Characteristics of the Impeller. Jiangsu University of Science and Technology, 2013.

[5] Zhang Lei, Yang Weibo, Liang Shoufang Review of fluid-solid interaction in turbomachinery. Thermal Power Generation, Vol. 12, 2014, p. 1-8.

[6] Li Jiafeng, Wang Jun, Li Hui, et al. Numerical analysis on aero elasticity of air-conditioning axial flow fan blade. Fan Technology, Vol. 6, 2015, p. 21-27.

[7] Hy Cui, Ding N., Hong M. Aero Elastic Analysis of Blades and Identification of Crack Damage. Dalian University of Technology, 2015.

[8] Ata I. Experimental validation of turbomachinery blade vibration predictions. European Spine Journal, Vol. 161, Issues 1-8, 2004, p. 1-21.

[9] Salhi B., Lardies J., Berthillier M. Identification of modal parameters and aero elastic coefficients in bladed disk assemblies. Mechanical Systems and Signal Processing, Vol. 23, Issue 6, 2009, p. 1894-1908.

[10] Fan Bonan, Zhang Yubo, Wang Haidou Research status for dynamic monitoring impellers' of blades based on blade tip-timing. Vibration and Shock, Vol. 5, 2016, p. 96-102.

[11] Meng Xianghai, Wang Jinquan, Liu Yuan, et al. Analysis of diesel engine sound signal based on correlation dimension. Internal Combustion Engine, Vol. 1, 2011, p. 56-59.

[12] Zhou Zhongning Research on Fluid-Solid Interaction Characteristic of Counter Rotation Axial Flow Fan and Its Application Based on Nonlinear Dynamics. China University of Mining and Technology, Xuzhou, 2009.

[13] Shang Wen Study on the Condition Monitoring and Fault Diagnosis of the Gas Turbine Blade. Beijing University of Chemical Technology, 2014.

[14] Yu Derong, Wei Qinglian, Wang Yongqiang Multidimensional cavitation monitoring system based on full-wave rectified spectrum analysis and its application. Journal of Wuhan University, Vol. 43, Issue 1, 2010, p. 112-115.

[15] Li Jiafeng, Wang Jun, Li Hui, Wen Rui Numerical analysis on aero elasticity of air-conditioning axial flow fan blade. Hua Zhong University of Science and Technology, Vol. 6, 2015, p. 26-28.

[16] Li Shaohui Fluid-Solid Coupling Features Analysis and Fault Diagnosis of Fan Blade. China University of Mining and Technology, 2009.

[17] Duan Daoqi, Fang Zhiqiang, Li Zhihua, Yu Yue Study on fiber-beam tip timing sensor for blade vibration detection. Collection and Academic Conference of China Optical Society, 2004.

[18] Zhang Xinming Nonlinear Filtering with Applications to Communication and Navigation System. Beijing University of Posts and Telecommunications, Beijing, 2012.

[19] Wang Fengli, Ma Xiaojiang Fault diagnosis of rotating machinery based on chaos. Journal of Dalian University of Technology, Vol. 9, 2003, p. 636-639.

[20] Zhao Jingxiao Detection and Circuit Realization of Weak Periodic Signal Based on Chaos Oscillator in Mechanical Fault Diagnosis. Hangzhou Dianzi University, Hangzhou, 2011. 
[21] Zhang Ruiqin, Weng Jiansheng Blade flutter analysis based on fluid solid coupling. Computer Simulation, Vol. 28, Issue 3, 2011, p. 48-51.

[22] Zhang Mingming, Zhang Wei, Hou Anping, et al. Progress and review of flip flutter research on impeller machinery. Progress in Mechanics, Vol. 41, Issue 1, 2011, p. 26-38.

[23] Chen Hai Xia, Qi Wen Jun, Wang Liang Ying, et al. Research on the fatigue properties of fluidsolid coupling of the wind turbine impeller. Mechanical Design and Manufacturing, Vol. 12, 2015, p. 31-34.

[24] Nagaoka S., Nakagabashi Y., Yagawa G. Parallelization of enriched free mesh method for large scale fluid-solid coupled analysis. Procedia Engineering, Vol. 90, 2014, p. 288-293.

[25] Wu Zheng Ren, Wang Song Ling, Rong Rui, Sun Zhe Dynamic characteristic analysis on impeller of centrifugal fan based on fluid-solid coupling. Journal of Chinese Society of Power Engineering, Vol. 1, 2013, p. 53-60.

[26] Zhu Shuhua, Tong Mingbo, Wang Yuequan Experiment and numerical simulation for bird impact on aircraft windshield. Chinese Journal of Applied Mechanics, Vol. 9, 2009, p. 451-456.

[27] Mao Yijun, Qi Datong, Liu Qiuhong Analysis of the aeroacoustics in centrifugal fan based on numerical simulation of unsteady viscous flow. Journal of Xian Jiaotong University, Vol. 39, Issue 9, 2005, p. 984-991.

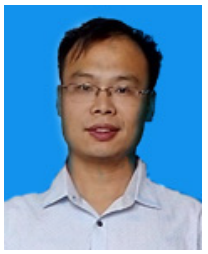

Chenxu Luo received Ph.D. degree in School of Mechatronic Engineering, China University of Mining and Technology, Xuzhou, China, in 2015. Now he works at Henan Polytechnic University. His current research interests include mining machinery and fault diagnosis.

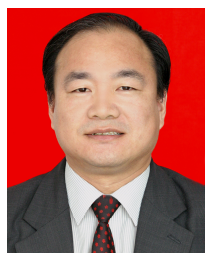

Shuangxi Jing received Ph.D. degree in School of Mechanical Electronic and Information Engineering, University of Ming and Technology, Beijing, China, in 2000. Now he works at Henan Polytechnic University. His current research interests include Mining machinery and fault diagnosis.

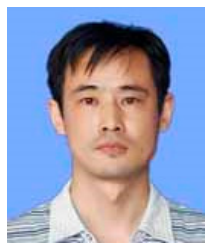

Zhiyan Wang received Ph.D. degree in School of Mechatronic Engineering, China University of Mining and Technology, Xuzhou, China, in 2008. Now he works at Henan Polytechnic University. His current research interests include signal processing and fault diagnosis.

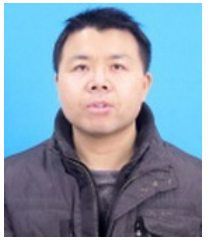

Junfa Leng received Ph.D. degree in School of Mechanical and Power Engineering, Henan Polytechnic University, Jiaozuo, China, in 2016. Now he works at Henan Polytechnic University. His current research interests include mechanical vibration and fault diagnosis. 